\title{
A purely Kerr nonlinear model admitting flat-top solitons
}

\author{
Liangwei Zeng ${ }^{1,2}$, Jianhua Zeng ${ }^{1,2,6}$, Yaroslav V. Kartashov ${ }^{3}$, and Boris A. Malomed ${ }^{4,51}$ \\ ${ }^{11}$ State Key Laboratory of Transient Optics and Photonics, \\ Xi'an Institute of Optics and Precision Mechanics of CAS, Xi'an 710119, China \\ ${ }^{2}$ University of Chinese Academy of Sciences, Beijing 100084, China \\ ${ }^{3}$ Institute of Spectroscopy, Russian Academy of Sciences, Troitsk, Moscow, 108840, Russia \\ ${ }^{4}$ Department of Physical Electronics, School of Electrical Engineering, \\ Faculty of Engineering, Tel Aviv University, Tel Aviv 69978, Israel \\ ${ }^{5}$ ITMO University, St. Petersburg 197101, Russia \\ ${ }^{6}$ Corresponding author: zengjh@opt.ac.cn
}

\begin{abstract}
We elaborate one- and two-dimensional (1D and 2D) models of media with self-repulsive cubic nonlinearity, whose local strength is subject to spatial modulation that admits the existence of flat-top solitons of various types, including fundamental ones, 1D multipoles, and 2D vortices. Previously, solitons of this type were only produced by models with competing nonlinearities. The present setting may be implemented in optics and Bose-Einstein condensates. The 1D version gives rise to an exact analytical solution for stable flat-top solitons, and generic families may be predicted by means of the Thomas-Fermi approximation. Stability of the obtained flat-top solitons is analyzed by means of linear-stability analysis and direct simulations. Fundamental solitons and 1D multipoles with $k=1$ and 2 nodes, as well as vortices with winding number $m=1$, are completely stable. For multipoles with $k \geq 3$ and vortices with $m \geq 2$, alternating stripes of stability and instability are identified in their parameter spaces.
\end{abstract}

The formation of bright spatial solitons in one-dimensional (1D) uniform media is a commonly known result of the balance between the diffraction and self-focusing nonlinearity [1]; however, making them stable in higher-dimensional media is a challenging issue owning to the presence of the wave collapse, which can, in an usual way, be suppressed with an aid of linear periodic potentials [1,2]. The situation may be different in physical settings with inhomogeneous strength of the local nonlinearity [2]. In optics, such settings may be engineered by means of properly designed photonic-crystal structures, with voids filled by solid [3] or liquid [4-6] materials with different values of the Kerr coefficient. Alternatively, one can use nonuniform distributions of nonlinearity-enhancing dopants [7, 8]. In Bose-Einstein condensates (BECs), similar nonlinearity landscapes can be created by means of the Feshbach resonance (FR) locally controlled by spatially nonuniform optical [9-11] or magnetic [12, 13] fields. In particular, it was predicted that self-defocusing nonlinearity, whose local strength grows from the center to periphery in the $D$ dimensional space, with radial coordinate $r$, at any rate faster than $r^{D}$, can support a great variety of robust self-trapped modes, including 1D fundamental, dipole and multipole solitons, 2D solitary vortices with arbitrarily high topological charge $m$ [14, 15], and sophisticated 3D modes, such as soliton gyroscopes [16] and skyrmions [17]. A characteristic feature of these localized modes is nonlinearizability of the underlying equations for their decaying tails, on the contrary to the usual bright solitons maintained by uniform self-focusing, whose exponential tails are produced by the corresponding linearized equations. The exploration of different kinds of bright solitons supported by this scheme has currently been extended to a variety of other physical settings [18]-[23].

The objective of this Letter is to demonstrate another natural setting, which gives rise to families of 1D and 2D flat-top solitons. This is a known variety of self-trapped modes, with a potential for applications [24], which are usually supported by systems with competing focusing and defocusing nonlinearities [25], such as cubic-quintic [26-29], cubic-quartic [30,31], and quadratic-cubic [32-34] combinations, as well as by cubic terms with an additional logarithmic factor $[33,35]$ (the two former types of the nonlinearity were recently realized experimentally in optics [36] and as "quantum droplets" BEC [37, 38], respectively). However, stable flat-top solitons were not previously found in physical models with the cubiconly nonlinearity, while here we demonstrate that this is possible, in 1D and 2D geometries alike, for various soliton species (fundamental, multipole, vortical), if the coefficient of the cubic defocusing is subjected to an appropriate spatial modulation. In addition to the systematically produced numerical results, we also obtain particular exact stable solutions for the 1D fundamental flat-top solitons, and develop the ThomasFermi (TF) approximation for generic soliton states and 2D vortices. Stability of all the solutions is investigated via the linear-stability analysis and numerical simulations.

The basic model is introduced as the scaled Schrödinger equation governing the evolution of the dimensionless complex amplitude of a light beam propagating in a cubic nonlinear medium, or the mean-field wave function in a BoseEinstein condensate (BEC), $\psi(\mathbf{r}, t)$ :

$$
i \psi_{t}=-(1 / 2) \nabla^{2} \psi+\sigma(r)|\psi|^{2} \psi
$$

which is written in the $2 \mathrm{D}$ form, with $\mathbf{r}=(x, y)$. Here $t$ is the evolution variable, representing time in the BEC version of the model, or the propagation distance in optics. The axially symmetric nonlinearity-modulation profile, $\sigma(r)>0$, is chosen in the form which readily helps to create flat-top modes of radius $r_{0}$,

$$
\sigma(r)=\left\{\begin{array}{c}
\sigma_{\mathrm{int}}, r \leq r_{0}, \\
\sigma_{\mathrm{ext}} \exp \left[\alpha\left(r+\alpha^{-1 / 2}-r_{0}\right)^{2}-1\right], r>r_{0},
\end{array}\right.
$$

with constants $\sigma_{\text {int,ext }}>0$ and $\alpha>0$. Note that Eq. (2) implies that $\sigma\left(r=r_{0}\right)=\sigma_{\text {ext }}$. The 1D version of the model 
corresponds to Eq. (1) with single coordinate $x$, while $r$ and $r_{0}$ are replaced by $|x|$ and $x_{0}>0$ in Eq. (2). This profile can be created by means of the above-mentioned methods - for instance, by the application of an FR-controlling magneticfield profile to the BEC layer, with a constant detuned value at $r<r_{0}$, and one approaching the exact resonant value at $r>$ $r_{0}$. It is relevant to mention that similar cylindrical optical-box potentials are used in current BEC experiments [39].

Wave functions of stationary states with real chemical potential $\mu$ (alias propagation constant $-\mu$ in terms of optics) and integer vorticity $m$ are sought for, in ploar coordinates $(r, \theta)$, as $\psi(\mathbf{r}, t)=w(r) \exp (i m \theta-i \mu t)$, with real $w$ determined by the equation

$$
\mu w=-(1 / 2)\left(w^{\prime \prime}+r^{-1} w^{\prime}-m^{2} r^{-2} w\right)+\sigma(r) w^{3}
$$

in $2 \mathrm{D}$, or its counterpart in $1 \mathrm{D}$. First, the $1 \mathrm{D}$ version of the model admits an exact solution, with $\mu=3 \alpha / 2$, $w\left(x \leq\left|x_{0}\right|\right)=\sqrt{3 \alpha / 2 \sigma_{\text {ext }}}$, and

$$
\begin{aligned}
& w\left(|x|>x_{0}\right)=\left(\alpha / \sqrt{2 \sigma_{\text {ext }}}\right)\left(|x|+\alpha^{-1 / 2}-x_{0}\right) \\
& \quad \times \exp \left(-(1 / 2)\left[\alpha\left(|x|+\alpha^{-1 / 2}-x_{0}\right)^{2}-1\right]\right) .
\end{aligned}
$$

Note that Eq. (4) gives $d w / d x\left(x=x_{0}\right)=0$, which is necessary for the continuity at $x=x_{0}$, while the remaining continuity condition for $w(x)$ imposes a relation on constants of modulation profile (2): $\sigma_{\text {int }}=3 \sigma_{\text {ext }}$. This profile is continuous in the limit case of $x_{0}=0$, for which exact solution (4) remains valid. Numerical solutions are displayed below for the continuous modulation profile with $\sigma_{\text {int }}=\sigma_{\text {ext }}$.

An analytical approximation for generic soliton shapes can be obtained in the Thomas-Fermi (TF) approximation, which neglects derivatives in Eq. (3) [40]:

$$
w_{\mathrm{TF}}^{2}=[\sigma(r)]^{-1}\left[\mu-m^{2} /\left(2 r^{2}\right)\right]
$$

at $r^{2}>m^{2} / 2 \mu$, and $w_{\mathrm{TF}}^{2}=0$ at $r^{2}<m^{2} / 2 \mu$. This approximation makes it possible to predict the dependence of the norm of the soliton family on the chemical potential,

$$
\begin{gathered}
N_{2 \mathrm{D}}=2 \pi \int_{0}^{\infty} w^{2}(r) d r \approx \pi \sigma_{\mathrm{int}}^{-1} \times \\
{\left[\mu r_{0}\left(r_{0}+\alpha^{-1 / 2}\right)-\left(m^{2} / 2\right) \ln \left(2 e \mu r_{0}^{2} / m^{2}\right)\right]}
\end{gathered}
$$

(here $e$ is the base of the natural logarithm), which is valid at $\mu>m^{2} /\left(2 r_{0}^{2}\right)$.

In the numerical form, stationary profiles of both 1D and 2D modes were found by means of the Newton's method applied to Eq. (3). The subsequent stability analysis was based on the usual ansatz, $\psi=\left[w(r)+p_{+}(r) \exp (i n \theta+\lambda t)+\right.$ $\left.p_{-}^{*}(r) \exp \left(-i n \theta+\lambda^{*} t\right)\right] \exp (i m \theta-i \mu t)$, where $p_{ \pm}(r)$ represent perturbation eigenmodes with eigenvalue $\lambda, *$ stands for the complex conjugate, and $n$ is an integer azimuthal index. Then, the eigenvalue problem amounts to the solution of linear equations,

$$
\begin{gathered}
i \lambda p_{ \pm}=\mp(1 / 2)\left[p_{+}^{\prime \prime}+r^{-1} p_{ \pm}^{\prime}-(m \pm n)^{2} r^{-2} p_{ \pm}\right] p \\
\mp \mu p_{ \pm}+\sigma w^{2}\left(2 p_{ \pm}+p_{\mp}\right),
\end{gathered}
$$
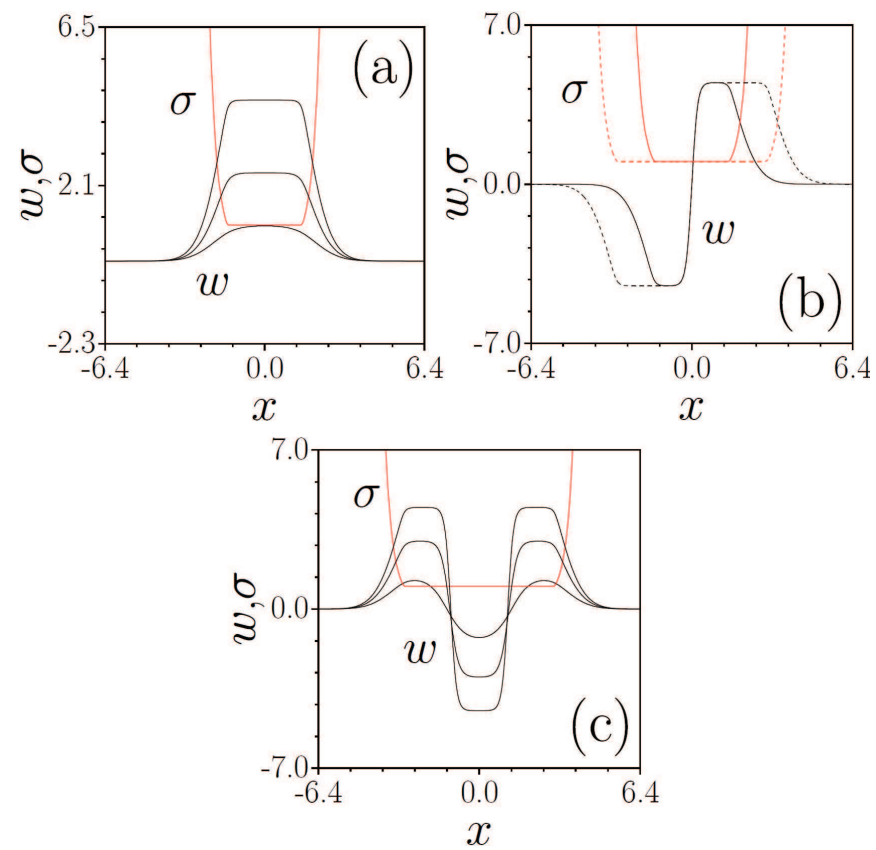

FIG. 1. (a) Fundamental flat-top $(k=0)$ solitons with $\mu=1,6,20$ at $x_{0}=1.5$. (b) Flat-top dipole $(k=1)$ solitons with $\mu=20$ at $x_{0}=$ 1.5 and 3 (solid and dashed lines, respectively) (c) The transition of tripole $(k=2)$ modes into the flat-top ones with increasing $\mu=$ 2,9 , and 20 at $x_{0}=3$. Here and in Fig. 4, red lines represent the nonlinearity-modulation profiles given by Eq. ( 2) with $\alpha=$ $\sigma_{\text {ext,int }}=1$ (these values are used throughout the work). All modes with $k=0,1$, and 2 are stable.
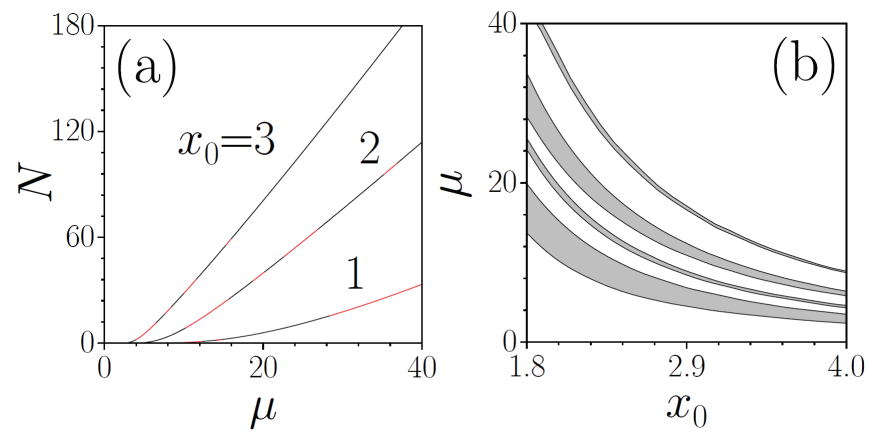

FIG. 2. (a) The norm versus $\mu$ for $1 \mathrm{D}$ multipoles with $k=6$ at different values of $x_{0}$. Here and in Fig. 5(a), stable and unstable segments are distinguished by the black and red colors, respectively. (b) Stability (white) and instability (gray) domains for the modes with $k=6$ in the $\left(x_{0}, \mu\right)$ plane.

or their $1 \mathrm{D}$ counterparts. In particular, the exact solution given by Eq. (4) is found to be always stable.

Typical profiles of 1D flat-top solitons with the number of nodes $k=0,1$, and 2 (fundamental, dipole, and tripole solitons, respectively) at different values of $\mu$ are displayed in Fig. 1, which clearly shows that the solitons' shape gets flatter with the increase of $\mu$. The functional form of soliton changes considerably as $\mu$ increases. For instance, solitons with large 


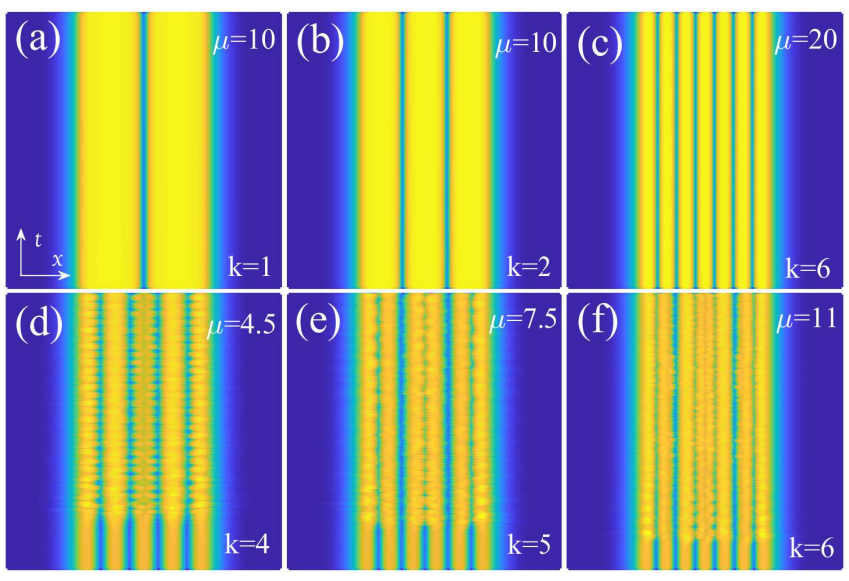

FIG. 3. Stable evolution of perturbed 1D solitons, for $x_{0}=3$ : (a) a dipole $(k=1)$ with $\mu=10$; (b) a tripole $(k=2)$ with $\mu=10$; (c) a multipole $(k=6)$ with $\mu=20$. The evolution of unstable multipoles, also for $x_{0}=3$ : (d) a $k=4$ with $\mu=4.5$; (e) $k=5$ with $\mu=7.5$; (f) $k=6$ with $\mu=11$. The evolution range is $0<t<1000$.
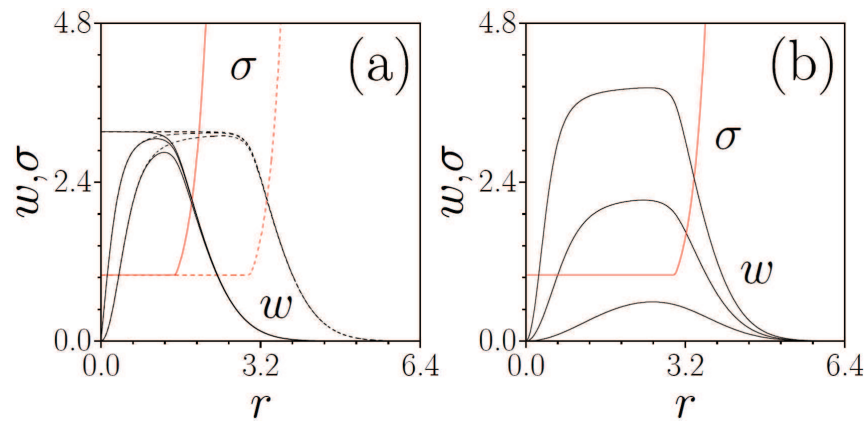

FIG. 4. (a) Profiles of $2 \mathrm{D}$ solitons with vorticities $m=0,1,2$ and $\mu=10$ at $r_{0}=1.5$ and 3 (solid and dashed lines, respectively). (b) The transition of vortices with $m=2$ into the flat-top shape with increasing $\mu=1,5,15$ at $r_{0}=3$.

number of nodes $k$ at small values of $\mu$ resemble trigonometric functions, while at large $\mu$ values they can be viewed as complexes of several well-localized dark solitons. Families of all solitons with $k \leq 2$ are completely stable (at least, up to $\mu=40$ ), while instability domains appear at $k \geq 3$. To illustrate this feature, Fig. 2(a) represents soliton families for $k=6$ by showing their norm vs. $\mu$ at several values of $x_{0}$. It is seen that even this high value of $k$, corresponding to "hashed" flat-top patterns, admits large stability segments, whose share increases with the growth of width $x_{0}$ of the modulation profile. Note also that all the $N(\mu)$ curves satisfy the "anti-Vakhitov-Kolokolov" criterion, $d \mu / d N>0$, which is a necessary condition for the stability of solitons in models with self-repulsive nonlinearities [41]. The alternating stability and instability domains for $k=6$ are charted in the $\left(x_{0}, \mu\right)$ plane in Fig. 2(b). Figure 2 shows that flat-top states are generally more stable than their more localized counterparts with the
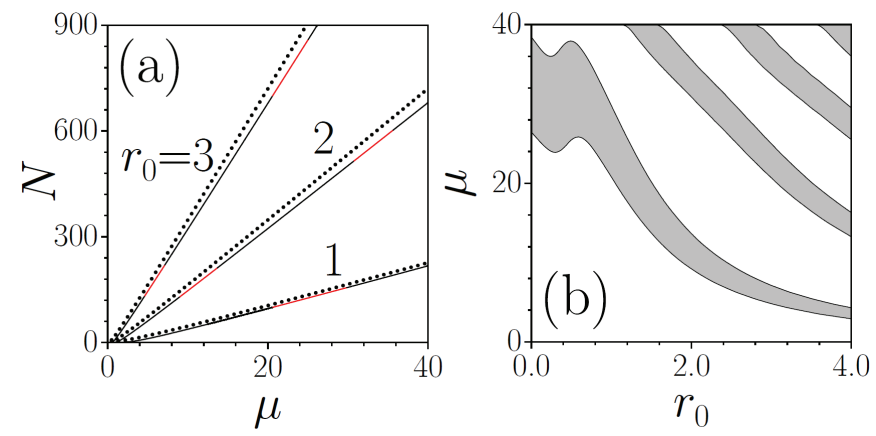

FIG. 5. (a) The norm of 2D double-vortex solitons $(m=2)$ versus $\mu$ at different values of width $r_{0}$ of the nonlinearity-modulation profile. Dotted lines display the prediction of the TF approximation, as per Eq. (6). (b) Stability (white) and instablility (gray) domains for the solitons with $m=2$ in the $\left(r_{0}, \mu\right)$ plane.

same number of nodes $k$.

Typical examples of the evolution of stable 1D flat-top solitons are displayed in Figs. 3(a)-(c), while evolution of their unstable counterparts is shown in Figs. 3(d-f). Unstable multipoles spontaneously develop oscillations, keeping the number of nodes.

The profiles of 2D solitons with vorticities $m=0$ (fundamental solitons), $m=1$, and $m=2$ for different values of $r_{0}$ are displayed in Fig. 4(a), which shows that the width of the flat-top solitons increases with $r_{0}$, similar to their $1 \mathrm{D}$ counterparts. Further, Fig. 4(b) displays profiles of the vortices with $m=2$ and different values of $\mu$, demonstrating that 2D solitons also get flatter with the increase of $\mu$.

Typical dependencies $N(\mu)$ for the vortex families with $m=2$ are displayed in Fig. 5(a), featuring a nearly linear form for all values of $r_{0}$, and considerable growth of $N$ with the increase of $r_{0}$. These features are well predicted by the TF approximation, as seen in the figure. The $2 \mathrm{D}$ modes with $m=0$ and 1 are completely stable, at least up to $\mu=40$, while the vortices with $m \geq 2$ demonstrate alternation of stability and instability domains in the $\left(r_{0}, \mu\right)$ plane in Fig. 5(b). Finally, the evolution of the flat-top 2D vortices is displayed in Fig. 6, demonstrating that those with $m=2$ or 3, which are unstable, split into persistently rotating pairs or triplets of unitary vortices.

In conclusion, we have demonstrated that families of stable flat-top solitons, including 1D multipoles and 2D vortices, can be created in media with cubic self-repulsive nonlinearity whose local strength is subject to an appropriate spatial modulation. To our knowledge, this model predicts the first stable flat-top solitons realized with the cubic-only nonlinearity, in contrast to previous results which demonstrated such modes solely in systems with competing attractive and repulsive nonlinearities, thus providing an alternative way to create and stabilize the flat-top modes with free intrinsic parameters. We have checked the stability of all the obtained flat-top solitons by means of the linear-stability analysis and direct simulations. Both the $1 \mathrm{D}$ and $2 \mathrm{D}$ solitons become flatter with the increase of their chemical potential. 1D multipoles with 

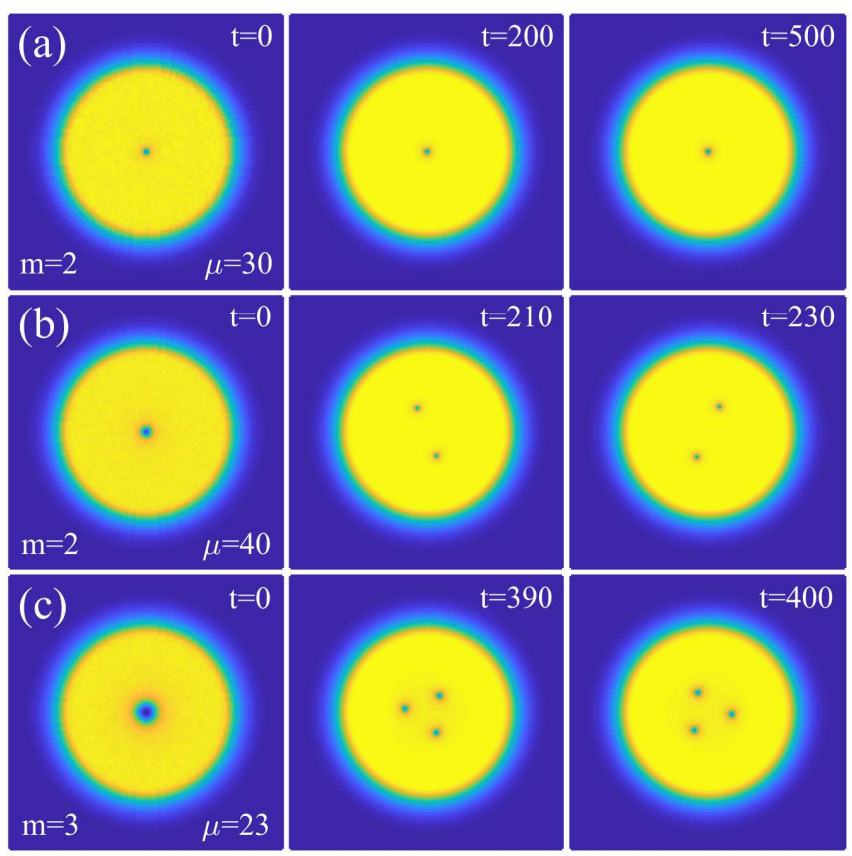

$k=0,1$, and 2 nodes, as well as $2 \mathrm{D}$ solitons with vorticities $m=0$ and 1 are completely stable, while higher-order modes, with $k \geq 3$ and $m \geq 2$, respectively, feature alternating stability and instability domains. Such self-trapped modes can be created in BEC and optics by means of available experimental techniques.

The work of LZ and JZ was supported by the NSFC (Nos. 61690224, 61690222), and by the Youth Innovation Promotion Association of the Chinese Academy of Sciences (No. 2016357). The work of B.A.M. was partly supported by the Israel Science Foundation through grant No. 1287/17.
FIG. 6. The evolution of perturbed $2 \mathrm{D}$ vortex solitons at $r_{0}=4$ : (a) a stable soliton with $m=2, \mu=30$; (b) an unstable soliton with $m=2, \mu=40$; (c) an unstable one with $m=3, \mu=23$.
[1] Y. S. Kivshar and G. P. Agrawal, "Optical Solitons: From Fibers to Photonic Crystals," (Academic, San Diego, CA, 2003).

[2] Y. V. Kartashov, B. A. Malomed, and L. Torner, Rev. Mod. Phys. 83, 247 (2011).

[3] F. Luan, A. K. George, T. D. Hedley, G. J. Pearce, D. M. Bird, J. C. Knight, and P. S. J. Russell, Opt. Lett. 29, 2369 (2004).

[4] A. Ferrando, M. Zacarés, P. Fernandez de Cordoba, D. Binosi, and J. A. Monsoriu, Opt. Express 11, 452 (2003).

[5] A. Fuerbach, P. Steinvurzel, J. A. Bolger, A. Nulsen, and B. J. Eggleton, Opt. Lett. 30, 830 (2005).

[6] P. Dalgaard Rasmussen, F. H. Bennet, D. N. Neshev, A. A. Sukhorukov, C. R. Rosberg, W. Krolikowski, O. Bang, and Y. S. Kivshar, Opt. Lett. 34, 295 (2009).

[7] J. Hukriede, D. Runde, and D. Kip, J. Phys. D 36, R1 (2003).

[8] A. Piccardi, A. Alberucci, N. Tabiryan and G. Assanto, Opt. Lett. 36, 1356 (2011).

[9] R. Yamazaki, S. Taie, S. Sugawa, and Y. Takahashi, Phys. Rev. Lett. 105, 050405 (2010).

[10] M. Yan, B. J. DeSalvo, B. Ramachandhran, H. Pu, and T. C. Killian, Phys. Rev. Lett. 110, 123201 (2013).

[11] L. W. Clark, L.-C. Ha, C.-Y. Xu, and C. Chin, Phys. Rev. Lett. 115, 155301 (2015).

[12] S. Ghanbari, T. D. Kieu, A. Sidorov, and P. Hannaford, J. Phys. B: At. Mol. Opt. Phys. 39, 847 (2006).

[13] O. Romero-Isart, C. Navau, A. Sanchez, P. Zoller, and J. I. Cirac, Phys. Rev. Lett. 111, 145304 (2013).

[14] O. V. Borovkova, Y. V. Kartashov, L. Torner, and B. A. Malomed, Phys. Rev. E 84, 035602(R) (2011).

[15] O. V. Borovkova, Y. V. Kartashov, B. A. Malomed, and L. Torner, Opt. Lett. 36, 3088 (2011).
[16] R. Driben, Y. V. Kartashov, B. A. Malomed, T. Meier, and L. Torner, Phys. Rev. Lett. 112, 020404 (2014).

[17] Y. V. Kartashov, B. A. Malomed, Y. Shnir, and L. Torner, Phys. Rev. Lett. 113, 264101 (2014).

[18] Q. Tian, L. Wu, Y. Zhang, and J.-F. Zhang, Phys. Rev. E 85, 056603 (2012).

[19] J. Zeng and B. A. Malomed, Phys. Rev. E 86, 036607 (2012).

[20] Y. Wu, Q. Xie, H. Zhong, L. Wen, and W. Hai, Phys. Rev. A 87, 055801 (2013).

[21] R. Driben, Y. V. Kartashov, B. A. Malomed, T. Meier, and L. Torner, New J. Phys. 16, 063035 (2014).

[22] D. Guo, J. Xiao, L. Gu, H. Ji, and L. Dong, Physica D 343, 1 (2017).

[23] J. Zeng and B. A. Malomed, Phys. Rev. E 95, 052214 (2017).

[24] M. A. Porras and F. Ramos, Opt. Lett. 42, 3275 (2017).

[25] Y. Kartashov, G. Astrakharchik, B. Malomed, and L. Torner, Nature Rev. Phys. in press.

[26] Kh. I. Pushkarov, D. I. Pushkarov, I. V. Tomov, Opt. Quant. Electr. 11, 471 (1979).

[27] M. Quiroga-Teixeiro and H. Michinel, J. Opt. Soc. Am. B 14, 2004 (1997).

[28] M. Quiroga-Teixeiro, A. Berntson, H. Michinel, J. Opt. Soc. Am. B 16, 1697 (1999).

[29] D. Mihalache, D. Mazilu, L.-C. Crasovan, I. Towers, A. V. Buryak, B. A. Malomed, L. Torner, J. P. Torres, and F. Lederer, Phys. Rev. Lett. 88, 073902 (2002).

[30] D. S. Petrov, Phys. Rev. Lett. 115, 155302 (2015).

[31] Y. V. Kartashov, B. A. Malomed, L. Tarruell, and L. Torner, Phys. Rev. A 98, 013612 (2018).

[32] A. V. Buryak, P. Di Trapani, D. V. Skryabin, and S. Trillo, Phys. Rep. 370, 63-235 (2002). 
[33] D. S. Petrov and G. E. Astrakharchik, Phys. Rev. Lett. 117, 100401 (2016).

[34] G. E. Astrakharchik and B. A. Malomed, Phys. Rev. A 98, 013631 (2018).

[35] Y. Li, Z. Luo, Y. Liu, Z. Chen, C. Huang, S. Fu, H. Tan, and B. A. Malomed, New J. Phys. 19, 113043 (2017).

[36] E. L. Falcão-Filho, C. B. de Araújo, G. Boudebs, H. Leblond, and V. Skarka, Phys. Rev. Lett. 110, 013901 (2013).

[37] C. R. Cabrera, L. Tanzi, J. Sanz, B. Naylor, P. Thomas, P. Cheiney, L. Tarruell, Science 359, 301-304 (2018).
[38] G. Semeghini, G. Ferioli, L. Masi, C. Mazzinghi, L. Wolswijk, F. Minardi, M. Modugno, G. Modugno, M. Inguscio, and M. Fattori, Phys. Rev. Lett. 120, 235301 (2018).

[39] R. Lopes, C. Eigen, N. Navon, D. Clement, R. P. Smith, and Z. Hadzibabic, Phys. Rev. Lett. 119, 190404 (2017).

[40] A. L. Fetter, Rev. Mod. Phys. 81, 647 (2009).

[41] H. Sakaguchi and B. A. Malomed, Phys. Rev. A 81, 013624 (2010).
[1] Y. S. Kivshar and G. P. Agrawal, "Optical Solitons: From Fibers to Photonic Crystals," (Academic, San Diego, CA, 2003).

[2] Y. V. Kartashov, B. A. Malomed, and L. Torner, "Solitons in nonlinear lattices," Rev. Mod. Phys. 83, 247-306 (2011).

[3] F. Luan, A. K. George, T. D. Hedley, G. J. Pearce, D. M. Bird, J. C. Knight, and P. S. J. Russell, "All-solid photonic bandgap fiber," Opt. Lett. 29, 2369-2371 (2004).

[4] A. Ferrando, M. Zacarés, P. Fernandez de Cordoba, D. Binosi, and J. A. Monsoriu, "Spatial soliton formation in photonic crystal fibers," Opt. Express 11, 452-459 (2003).

[5] A. Fuerbach, P. Steinvurzel, J. A. Bolger, A. Nulsen, and B. J. Eggleton, "Nonlinear propagation effects in antiresonant highindex inclusion photonic crystal fibers," Opt. Lett. 30, 830-832 (2005).

[6] P. Dalgaard Rasmussen, F. H. Bennet, D. N. Neshev, A. A. Sukhorukov, C. R. Rosberg, W. Krolikowski, O. Bang, and Y. S. Kivshar, "Observation of two-dimensional nonlocal gap solitons," Opt. Lett. 34, 295-297 (2009).

[7] J. Hukriede, D. Runde, and D. Kip, "Fabrication and application of holographic Bragg gratings in lithium niobate channel waveguides," J. Phys. D 36, R1-R16 (2003).

[8] A. Piccardi, A. Alberucci, N. Tabiryan and G. Assanto, "Dark nematicons," Opt. Lett. 36, 1356-1358 (2011).

[9] R. Yamazaki, S. Taie, S. Sugawa, and Y. Takahashi, "Submicron spatial modulation of an interatomic interaction in a BoseEinstein condensate," Phys. Rev. Lett. 105, 050405 (2010).

[10] M. Yan, B. J. DeSalvo, B. Ramachandhran, H. Pu, and T. C. Killian, "Controlling condensate collapse and expansion with an optical Feshbach resonance," Phys. Rev. Lett. 110, 123201 (2013).

[11] L. W. Clark, L.-C. Ha, C.-Y. Xu, and C. Chin, "Quantum Dynamics with Spatiotemporal Control of Interactions in a Stable Bose-Einstein Condensate," Phys. Rev. Lett. 115, 155301 (2015).

[12] S. Ghanbari, T. D. Kieu, A. Sidorov, and P. Hannaford, "Permanent magnetic lattices for ultracold atoms and quantum degenerate gases," J. Phys. B: At. Mol. Opt. Phys. 39, 847-860 (2006).

[13] O. Romero-Isart, C. Navau, A. Sanchez, P. Zoller, and J. I. Cirac, "Superconducting vortex lattices for ultracold atoms," Phys. Rev. Lett. 111, 145304 (2013).

[14] O. V. Borovkova, Y. V. Kartashov, L. Torner, and B. A. Malomed, "Bright solitons from defocusing nonlinearities," Phys. Rev. E 84, 035602(R) (2011).

[15] O. V. Borovkova, Y. V. Kartashov, B. A. Malomed, and L. Torner, "Algebraic bright and vortex solitons in defocusing media," Opt. Lett. 36, 3088 (2011).

[16] R. Driben, Y. V. Kartashov, B. A. Malomed, T. Meier, and L.
Torner, "Soliton Gyroscopes in Media with Spatially Growing Repulsive Nonlinearity,” Phys. Rev. Lett. 112, 020404 (2014).

[17] Y. V. Kartashov, B. A. Malomed, Y. Shnir, and L. Torner, "Soliton gyroscopes in media with spatially growing repulsive nonlinearity," Phys. Rev. Lett. 113, 264101 (2014).

[18] Q. Tian, L. Wu, Y. Zhang, and J.-F. Zhang, "Vortex solitons in defocusing media with spatially inhomogeneous nonlinearity," Phys. Rev. E 85, 056603 (2012).

[19] J. Zeng and B. A. Malomed, "Bright solitons in defocusing media with spatial modulation of the quintic nonlinearity," Phys. Rev. E 86, 036607 (2012).

[20] Y. Wu, Q. Xie, H. Zhong, L. Wen, and W. Hai, "Algebraic bright and vortex solitons in self-defocusing media with spatially inhomogeneous nonlinearity," Phys. Rev. A 87, 055801 (2013).

[21] R. Driben, Y. V. Kartashov, B. A. Malomed, T. Meier, and L. Torner, "Three-dimensional hybrid vortex solitons," New J. Phys. 16, 063035 (2014).

[22] D. Guo, J. Xiao, L. Gu, H. Ji, and L. Dong, "One- and twodimensional bright solitons in inhomogeneous defocusing nonlinearities with an antisymmetric periodic gain and loss," Physica D 343, 1-6 (2017).

[23] J. Zeng and B. A. Malomed, "Localized dark solitons and vortices in defocusing media with spatially inhomogeneous nonlinearity," Phys. Rev. E 95, 052214 (2017).

[24] M. A. Porras and F. Ramos, "Quasi-ideal dynamics of vortex solitons embedded in flattop nonlinear Bessel beams", Opt. Lett. 42 , 3275-3278 (2017).

[25] Y. Kartashov, G. Astrakharchik, B. Malomed, and L. Torner, "Frontiers in multidimensional self-trapping of nonlinear fields and matter," Nature Rev. Phys. in press.

[26] Kh. I. Pushkarov, D. I. Pushkarov, I. V. Tomov, "Self-action of light beams in nonlinear media: soliton solutions." Opt. Quant. Electr. 11, 471-478 (1979).

[27] M. Quiroga-Teixeiro and H. Michinel, "Stable azimuthal stationary state in quintic nonlinear optical media," J. Opt. Soc. Am. B 14, 2004-2009 (1997).

[28] M. Quiroga-Teixeiro, A. Berntson, H. Michinel, "Internal dynamics of nonlinear beams in their ground states: short- and long-lived excitation,” J. Opt. Soc. Am. B 16, 1697-1704 (1999).

[29] D. Mihalache, D. Mazilu, L.-C. Crasovan, I. Towers, A. V. Buryak, B. A. Malomed, L. Torner, J. P. Torres, and F. Lederer, "Stable spinning optical solitons in three dimensions," Phys. Rev. Lett. 88, 073902 (2002).

[30] D. S. Petrov, "Quantum mechanical stabilization of a collapsing Bose-Bose mixture," Phys. Rev. Lett. 115, 155302 (2015).

[31] Y. V. Kartashov, B. A. Malomed, L. Tarruell, and L. Torner, "Three-dimensional droplets of swirling superfluids," Phys. Rev. A 98, 013612 (2018). 
[32] A. V. Buryak, P. Di Trapani, D. V. Skryabin, and S. Trillo, "Optical solitons due to quadratic nonlinearities: from basic physics to futuristic applications", Phys. Rep. 370, 63-235 (2002).

[33] D. S. Petrov and G. E. Astrakharchik, "Ultradilute lowdimensional liquids," Phys. Rev. Lett. 117, 100401 (2016).

[34] G. E. Astrakharchik and B. A. Malomed, "Dynamics of onedimensional quantum droplets," Phys. Rev. A 98, 013631 (2018).

[35] Y. Li, Z. Luo, Y. Liu, Z. Chen, C. Huang, S. Fu, H. Tan, and B. A. Malomed, "Two-dimensional solitons and quantum droplets supported by competing self- and cross-interactions in spinorbit-coupled condensates," New J. Phys. 19, 113043 (2017).

[36] E. L. Falcão-Filho, C. B. de Araújo, G. Boudebs, H. Leblond, and V. Skarka, "Robust two-dimensional spatial solitons in liquid carbon disulfide," Phys. Rev. Lett. 110, 013901 (2013).
[37] C. R. Cabrera, L. Tanzi, J. Sanz, B. Naylor, P. Thomas, P. Cheiney, L. Tarruell, "Quan-tum liquid droplets in a mixture of Bose-Einstein condensates," Science 359 , 301-304 (2018).

[38] G. Semeghini, G. Ferioli, L. Masi, C. Mazzinghi, L. Wolswijk, F. Minardi, M. Modugno, G. Modugno, M. Inguscio, and M. Fattori, "Self-bound quantum droplets in atomic mixtures," Phys. Rev. Lett. 120, 235301 (2018).

[39] R. Lopes, C. Eigen, N. Navon, D. Clement, R. P. Smith, and Z. Hadzibabic, "Quantum depletion of a homogeneous BoseEinstein condensate," Phys. Rev. Lett. 119, 190404 (2017).

[40] A. L. Fetter, "Rotating trapped Bose-Einstein condensates," Rev. Mod. Phys. 81, 647-691 (2009).

[41] H. Sakaguchi and B. A. Malomed, "Solitons in combined linear and nonlinear lattice potentials," Phys. Rev. A 81, 013624 (2010). 